Response to Commentaries on The Case of "Hiro": Treating Tourette Syndrome by Comprehensive Behavioral Intervention for Tics (CBIT)

\title{
Mechanical/Algorithmic Versus Flexible/Creative Clinical Practice: How Underlying Principles Bridge the Gap
}

\author{
JEREMY D. LICHTMAN ${ }^{\mathrm{a}, \mathrm{b}}$ \\ ${ }^{\text {a }}$ Graduate School of Applied \& Professional Psychology, Rutgers University-New Brunswick \\ ${ }^{b}$ Correspondence regarding this article should be sent to Jeremy Lichtman, Tourette Syndrome Clinic, Graduate \\ School of Applied \& Professional Psychology, 152 Frelinghuysen Road, Piscataway, NJ 08854 \\ Email: jdlichtman@gmail.com
}

\begin{abstract}
In the case of "Hiro" (Lichtman, 2017) I described the successful use of Comprehensive Behavioral Intervention for Tics (CBIT) in a young boy with Tourette Syndrome (TS). In three different commentaries on this case, authors noted important concepts regarding the use of manuals in treating TS; how an understanding of underlying principles is important in flexible use of manuals; and how training impacts the treatment of TS. In my response to these commentaries, I hope to continue the conversation of how to use manuals in psychotherapy in general as well as in the treatment of TS in particular. Specifically, I hope to address the seeming dichotomy between a mechanical, algorithmic approach to treatment versus a flexible, creative approach, and to ultimately show that an understanding of the underlying principles guiding manualized treatment provides room for ingenuity that proves this dichotomy false.

Key words: Tourette Syndrome; Tics Disorders; Comprehensive Behavioral Intervention for Tics (CBIT); Habit Reversal Training (HRT); cognitive-behavioral training (CBT); Attention Deficit Hyperactivity Disorder (ADHD); manualized therapy; individualized case formulation; case studies; clinical case studies

I would like to begin this article by thanking the five commentators for taking the time to share their insightful commentaries on the Case of Hiro. Specifically, I would like to thank Dr. Matthew Capriotti (2017) for his discussion on the role of treatment manuals in evidence-based treatment (EBT), providing an alternative approach to TS treatment when client motivation is low, and his fascinating ideas regarding the dichotomy and overlap between a "protocols-first" and a "principles-first" approach to therapy. I would also like to thank Ms. Brianna Wellen and Dr. Michael Himle (2017) for their conversation and insights regarding the importance of theory as well as general strong clinical skills when treating clients with TS, and their wonderful metaphor of the difference between a novice cook and expert culinarian. Finally, I would like to thank Dr. Daniela Colognori and Dr. Lori Rockmore (2017) for their commentary on the importance of training in the treatment of TS. They provide a roadmap for how they have
\end{abstract}


accomplished this at the Tourette Syndrome (TS) Program at Rutgers University and use the Case of Hiro as an example of the challenges faced by novice clinicians treating TS and the strengths of the Rutgers' TS program in training clinicians to overcome these.

Tourette Syndrome (TS) is a neurological disorder characterized by involuntary motor and vocal tics (Bitsko et al., 2014). While tics are neurobiological in origin, from a behavioral perspective (Capriotti \& Woods, 2013), they are maintained by a negative reinforcement cycle in which the individual with TS feels a discomfort around the area of the tic (called the "premonitory urge"), which feels like it can only be alleviated by performance of the tic behavior. Every time the tic behavior is performed, it reinforces the cycle by which the tics are maintained. Additionally, the presentations of TS can be incredibly idiosyncratic, with the range of tics, their severity, their frequency, and the multitude of comorbidities creating a vastly different presentation by any given individual with TS.

Given the social, emotional, and functional impairment that can be caused by tics, it is important that a treatment opportunity exists. To that end, Comprehensive Behavioral Intervention for Tics (CBIT), a form of Cognitive Behavioral Therapy (CBT), was developed based on the behavioral model described above (Woods et al., 2008) to provide individuals with TS the skills to manage their tics, and has been shown to be an efficacious evidence-based treatment (Cook \& Blacher, 2007; Piacentini et al., 2010). Knowing that CBIT has the randomized control trial (RCT) data proving that it is a successful form of treatment on a large scale, in the Case of Hiro I wanted to zero in and show how it could be successful on a small scale, with a single individual. More specifically, my goals in writing the Case of Hiro were to "analyze the clinical application of [CBIT] treatment for [TS]; to record and analyze how this application led to a successful treatment of TS-specifically, in what ways adherence to the manual was effective and what contravention from the manual was necessary-and to record the growth of a novice practitioner and analyze what I needed to learn to successfully treat my client” (Lichtman, 2015, p. 2).

In this article I will take the opportunity to revisit the Case of Hiro in light of the three commentaries written in response to the case. Specifically, I plan to use the Case of Hiro and the three commentaries to address a few particular questions that stood out to me when reading the commentaries. The first is, are manuals useful, and if they are, how so? This question will relate to Capriotti's (2017) article and the ideas that he brings up regarding "flexibility within fidelity" (also see Kendall, Gosch, Furr, \& Sood, 2008) as well as his insights regarding "Protocols First” treatment versus "Principles First” treatment.

Secondly, how important is understanding the underlying principles of a treatment, and how does that affect treatment? This question relates to Wellen and Himle's (2017) article regarding the importance of the underlying principles of CBT when using CBT treatments. Additionally, these first two questions more broadly relate to the dichotomy between what I would categorize as a mechanical/algorithmic approach versus a flexible/creative approach to psychotherapy.

Finally, based partly on the answers to these first two questions, how does one apply the fairly mechanized, algorithmic treatment component of Habit Reversal Therapy (HRT) to 
something as idiosyncratic as TS? My goal in writing this article is to find a middle ground between the mechanical/algorithmic and the flexible/creative approach to psychotherapy, as opposed to choosing one over the other. In this middle ground, maintaining fidelity with the "tried and true principles" (Wellen \& Himle, 2017) together with the benefits of experience can inform the intuition and creativity of the practitioner to best be able to help their clients live meaningful lives.

\section{THE IMPORTANCE OF MANUALS}

Before I delve into the question of the usefulness of manuals, it is important to discuss some of the findings in the field of decision making. This is an important detour because clinicians make decisions and judgments that affect the treatments they decide to provide and how they provide them. Pioneered by the psychologists Daniel Kahneman and Amos Tversky in the early 1970s, this field has found that human judgment consistently fails in systematic ways, largely because the human mind habitually falls prey to a number of biases and heuristics that inhibit the ability to think rationally about a decision (Kahneman, 2013). Examples of these biases include the fundamental attribution bias (overestimating our own role in situations while underestimating others; Ross, 1977); confirmation bias (searching for evidence to support one's own preconceptions; Nickerson, 1998); and anchoring (relying too much on information that was presented earlier; Tversky \& Kahneman, 1974), to name just a few.

The research shows that these biases have significant, real-world effects on human decision-making processes. For example, Nickerson (1998) argues that it was the confirmation bias that led to the reliance on tradition and logic in medicine, to the exclusion of experimentation and evidence, up until the $16^{\text {th }}$ century; and that this same bias has led to a continued lack of empiricism in current pseudo-medicine. He explains that physicians in the medieval century would see the cases that improved and, under the influence of the confirmation bias, would see those improvements as due to their interventions, as opposed to the natural progression of the illness; similarly, those who believe in pseudo-medicine will rely on the testimony of the successes of their craft and the failure of modern medicine to the exclusion of modern research trials.

In the field of clinical psychology these biases can lead to misdiagnoses, poor case formulations, and subpar treatment options (Garb, 2005). When it comes to diagnoses, for example, clinicians may often "satisfice” (Kleinmuntz, 1990) — that is, rely on information based on prior expectations or even their own confidence, and therefore end up not asking relevant questions - instead of using reliable, standardized, diagnostic tools and criteria (Garb, 2005). When it comes to treatment, cognitive biases can lead to the overreliance on one's own impressions of success (e.g., confirmation bias, "The client says they feel better, so I must be doing something right”) and therefore ignoring other important treatment items. An example of this would be a therapist who uses CBT to treat anxiety, but relies solely on cognitive work and ignores the use of exposures. Schulte, Kunzel, Pepping, and Schulte-Bahrenberg (1992) showed that when clinicians (experienced or otherwise) break from standardization in the treatment of specific phobias, their adaptations tend to lead to less advantageous treatment outcomes when compared to clinicians who maintained strict fidelity to a standardized treatment protocol. As 
Wilson (2007, 1998a) notes, these clinicians relied on their intuition, which is easily prey to biases (e.g., the availability heuristic, or only remembering the times that breaking from standardization succeeded), which can lead to less effective clinical practice.

To combat the common errors in human judgment, a method called "Statistical Prediction Rules” (SPR; Bishop \& Trout, 2004; Dawes, 2002; Grove \& Meehl, 1996) has been developed. An SPR is a mechanical formula (actuarial or algorithmic model) that, when based on the same evidence and compared to human experts, can outperform human judgment in almost any area (e.g., Bishop \& Trout, 2002; Dawes, 2002). As Bishop and Trout (2002) state, "SPRs have been proven more reliable than humans at predicting the success of electroshock therapy, criminal recidivism, psychosis and neurosis on the basis of MMPI profiles, academic performance, progressive brain dysfunction, the presence, location and cause of brain damage, and proneness to violence” (p. S198). Additionally, an SPR doesn't need to come in the form of a complex equation, but can be as simple as a checklist (Gawande, 2010). An example of a simple SPR checklist that has been responsible for saving an untold number of infant lives is the "Apgar" score; this test is performed by doctors immediately after birth, looks at five areas of infant functioning, and scores them on a scale of 1-3, with a low score indicating that the baby needs immediate medical attention (Gawande, 2010).

Explanations have been suggested for why mechanical procedures outperform clinical judgment. These include first, that these procedures are not influenced by external factors outside of the data itself (Dawes, Faust, Meehl, 1989); and second, that humans have difficulties with discovering the right information to use, attending to more than a few cues at a time, and appropriately weighting these cues (Bishop \& Trout, 2004). The implication for psychological practice is that an overreliance on one's own intuition in treatment, especially when there is good evidence-based practice in existence, can lead to ignoring important treatment factors and poor treatment outcome (Wilson, 2007). Manuals can be used to bypass these biases by automatically targeting what clinicians should do; providing a sequential order for providing treatment; not overloading the limited memory and attention capacity by which all humans are bound; and providing methods for monitoring progress (Bishop \& Trout, 2004; Wilson, 2007; Wilson 1998a; Wilson, 1998b). For all these reasons, it would certainly seem safe to say that, when manuals exist with a robust evidence-base behind them, they should be used as the first line of action.

However, what does one do in a case in which sticking with the manual does not seem to be providing the success that the evidence base suggests it should? Here again Wilson (2007) proves invaluable when he explains that the clinician needs to match the treatment of particular patients to the mechanisms that are maintaining the symptomology, and to utilize the treatment components that target those mechanisms. The idea is that by performing thorough functional analyses of symptomology and monitoring progress (inherent components of all CBT treatment protocols), the skilled clinician will be able to identify the causal mechanisms of the presenting problems and marry the most relevant elements of the treatment to those symptoms. Additionally, the clinician needs to take into account the client's adherence to the treatment assignments, and may need to allow for flexibility in order to maintain rapport and motivation, an important component of treatment adherence (Loeb et al., 2005). I believe this is the sort of flexibility I exhibited in my treatment of Hiro when I moved my focus more primarily from the 
environmental interventions component to the more behaviorally focused Habit Reversal Training (HRT) component; and when I adopted Hiro's interests into the treatment, e.g., by using video game characters as metaphors for his tics and his competing responses.

A final thought regarding Dr. Capriotti's distinction between Protocol First and Principle First treatment and my adherence to a Protocol First approach. I do believe there is a lot of merit to taking a Principle First approach and its emphasis on transdiagnostic underlying principles, and indeed there may be many situations where a Principle First approach would be recommended. However, as Wilson (2007) notes,

[One] limitation of relying only on principles is that it may miss the rich clinical content and context of treatment manuals. Moreover, in a related fashion, it might also miss specific maintaining mechanisms of different clinical disorders. This would be a problem especially for the mental health provider with less training in the treatment or lacking specialization in the target disorder (pp. 123-124).

\section{THE UTILITY OF UNDERSTANDING TREATMENT PRINCIPLES}

Before I begin answering the second question, I would like to expound briefly on . Colognori and Rockmore's (2017) discussion of the training at the Rutgers TS program. Beyond just training clinicians in the specialized skills required for treating TS and its common comorbidities, the training emphasizes understanding the principles behind why CBT is effective for the common conditions treated in the program. This has been my experience under the supervision of Dr. Shawn Ewbank when I was a practicum student in the program for two years, as well as currently as a postdoctoral fellow in the program. This training has provided me with the skills to understand, on a fundamental level, how to use new CBT manuals that I might encounter, as well as those skills needed to approach these manuals flexibly.

Wellen and Himle (2017) thoroughly cover the importance of the underlying principles in the Comprehensive Behavioral Intervention for Tics (CBIT) model in their response to the Case of Hiro, which means there is not much more to say here. However, I would like to add a few comments. While strict use of manuals will lead, on average, to more clinicians providing better treatment since treatment decisions will otherwise be too influenced by biases, Wilson (2007) does note that for more complex cases, expertise in underlying principles is important for flexibly adapting the protocols for success. Furthermore, he notes that clinicians must use knowledge of the underlying principles in order to target specific symptoms, as opposed to strictly matching the manual to the clients' DSM diagnoses (which he notes as a flaw in the standard practice of treatment-manual development today). Thus, an understanding of the principles is helpful inasmuch as it allows for informed clinical flexibility.

Additionally, there is some research that suggests greater therapist competence, which can be defined as greater knowledge of underlying principles, can lead to better psychotherapy outcomes. For example, a study by James, Blackburn, Milne, and Reichfelt (2001) demonstrates that, according to the Cognitive Therapy Scale (CTS-R), greater knowledge and experience of Cognitive Therapy (CT) leads to greater competence and therapeutic gains. 
What I hope to have demonstrated so far is that adherence to manualized treatments is a powerful and helpful means for bypassing human judgment errors (Kahneman, 2011; Wilson, 2007) by providing a proven, actuarial approach to improving therapy. That being said, having a strong understanding of the underlying principles supporting the manual's success allows for the flexibility and creativity needed to treat complex and/or treatment-resistant cases. So rather than breaking down psychotherapy into either a mechanical/algorithmic or a flexible/creative approach, I believe it is possible that a strong understanding of the principles and mechanisms behind an algorithmic approach in fact lends itself to creative use of these principles when necessary to bolster treatment effects.

\section{HRT: APPLYING AN ALGORITHMIC TREATMENT TO IDIOSYNCRATIC SYMPTOMOLOGY}

Up to now I have made the argument that, for symptoms where manuals exist, using a manual should be the go-to approach to begin treatment-essentially, taking an actuarial approach. However, I have also tried to stress the idea that following a manual in a cookie-cutter way is not always the ideal approach, especially for complex and/or treatment-resistant cases. In those cases, I have emphasized the importance of understanding the underlying principles and how that understanding leads to creativity and ingenuity in treatment. So what does this all mean for the treatment of TS?

Obviously, I began my treatment of Hiro from a manualized, Protocol First perspective. As treatment progressed, I deemphasized the elements that were less useful in treatment and emphasized using the more effective components. It is important to recognize, as Wellen and Himle (2017) noted, that some parts of the manual were less relevant for the successful treatment of Hiro, a fact that I incorporated into my decision to shift the focus of treatment. The decision was guided by the treatment team's understanding of the most important elements of treatment from a principled perspective. How the decision was made is tied to the idea of creativity and ingenuity in treatment-being flexible with a manual based on a thorough understanding of what makes the manual effective in the first place.

HRT is the component that received the most attention. HRT itself is incredibly algorithmic. Thus, even without following the CBIT manual, HRT is a highly structured form of therapy that requires fidelity to be successful (Woods et al., 2008). The structure of HRT does not vary, and for good reason: Without awareness (the first component of HRT), the client cannot develop an effective competing response (CR); without an effective CR, habituation won’t occur, and habituation is the key to successful tic management (Specht et al., 2013). However, tics can be incredibly idiosyncratic, with one individual's tics looking incredibly different from another's. This leads to two questions: (a) How can such a strictly-structured treatment lead to success with such widely varying symptomology, and (b) How are creativity and clinical judgment factored into using HRT?

Regarding the first question, one must recognize that despite the differences in tic presentation, the maintaining mechanisms behind these tics are the same (Verdellen et al., 2008). 
Therefore, regardless of which tics are present, the goal is always to reach habituation. Habituation is reached when the client is given enough time to not tic (e.g., to perform a competing response) while being aware of the decrease in urge to tic over that period of time. This is true regardless of whether the tic is a motor tic or a phonic tic, whether it is complex or simple. This doesn't mean that other factors of the client (e.g., motivation), or the client-therapist relationship (e.g., trusting), are not important, but the individual presentation of the tics themselves does not change this core element of treatment.

In terms of the second question, creativity and clinical judgment play a role in HRT when attending to the other factors mentioned above. Beyond the manual itself, successful treatment of TS requires a strong therapeutic relationship; client motivation; buy-in from the client; and so forth. These are the areas in which creativity and clinical judgment are not only helpful, but vital. For example, a clinician may use these tools within HRT to explain habituation to the client in a way that the client will understand and identify with. When I used video game analogies with Hiro to explain the habituation process, I was making decisions about how to make the treatment meaningful to Hiro, something that HRT itself does not explain how to do.

Alternatively, my decision to switch a CR that was not proving effective was another example; this was an important, flexible move based on the clinical judgment that Hiro's comfort was critical in order for him to continue using it and for habituation to occur.

A final example from the case of Hiro is when I decided to use imagery to bolster the strength of a competing response. In that case, I employed creativity within the structure of HRT. Thus, the visualizations inspired by video games were a way to bolster Hiro's motivation while simultaneously increasing his awareness of the urge to tic and the subsequent habituation to the urge as his use of the CR continued. All of these are examples of how, under the supervision of a TS expert supervisor, I was able to use an understanding of how tics function to creatively adapt HRT, an incredibly structured treatment, to the needs of my unique client, Hiro.

\section{PUTTING THE PIECES TOGETHER}

The commentaries written in response to the Case of Hiro allowed me to reflect upon the treatment of TS, as well as what enabled success when treating Hiro. This reflection allowed me to further appreciate the importance of manualized treatment in psychotherapy; the significance of understanding the underlying principles that make these manuals successful; and how that understanding enhances the use of structured, algorithmic treatment protocols when working with clients both with TS and in general. When applying the lessons derived from the field of decision making, it becomes clear that a manualized treatment provides the best means of overcoming the natural human biases that can negatively influence the course of treatment.

Through the support and training of the Tourette Syndrome program at Rutgers, I was able in the Case of Hiro to develop a thorough understanding of the underlying principles of CBT in general, and CBIT in particular. This understanding allowed me to incorporate judicious flexibility when necessary, which in turn led to the successful treatment of Hiro and my development from a novice level trainee to a more experienced clinician. Ultimately, I have 
found that my clinical training in using a manual in the treatment of TS is a crucial starting point, but that clinical judgment based on a thorough understanding of the principles upon which the manual was founded is equally important, in order to allow for flexibility and creativity as needed.

\section{REFERENCES}

Bishop, M. A., \& Trout, J. D. (2002). 50 years of successful predictive modeling should be enough: Lessons for philosophy of science. Philosophy of Science, 69(S3), S197-S208.

Bishop, M. A., \& Trout, J. D. (2004). Epistemology and the psychology of human judgment. Oxford University Press.

Bitsko, R. H., Holbrook, J. R., Visser, S. N., Mink, J. W., Zinner, S. H., Ghandour, R. M., \& Blumberg, S. J. (2014). A national profile of Tourette syndrome, 20112012. Journal of Developmental \& Behavioral Pediatrics,35(5), 317-322.

Capriotti, M.R. (2017). On protocols and principles in the Case of Hiro. Pragmatic Case Studies in Psychotherapy, 13(1), Article 2, 38-50. Available: http://pcsp.libraries.rutgers.edu

Capriotti, M.R., \& Woods, D. W. (2013). Cognitive-behavioral treatment for tics. In D. Martino \& J. F. Leckman (Eds.), Tourette syndrome (pp. 503-523). Oxford: Oxford University Press.

Colognori, D., \& Rockmore, L. (2017). Perspectives on training clinicians to effectively implement evidence-based treatment for Tourette Syndrome. Pragmatic Case Studies in Psychotherapy, 13(1), Article 4, 63-72. Available: http://pcsp.libraries.rutgers.edu

Cook, C. R., \& Blacher, J. (2007). Evidence-Based Psychosocial Treatments for Tic Disorders. Clinical Psychology: Science and Practice, 14(3), 252-267. doi:10.1111/j.1468-2850.2007.00085.x

Dawes, R. M. (2002). The ethics of using or not using statistical prediction rules in psychological practice and related consulting activities. Philosophy of Science, 69(S3), S178-S184.

Dawes, R. M., Faust, D., \& Meehl, P. E. (1989). Clinical versus actuarial judgment. Science, 243(4899), 1668-1674.

Garb, H. N. (2005). Clinical judgment and decision making. Annual Review of Clinical Psychology, 1, 67-89.

Gawande, A. (2010). The checklist manifesto: How to get things right. London: Profile Books.

Grove, W. M., \& Meehl, P. E. (1996). Comparative efficiency of informal (subjective, impressionistic) and formal (mechanical, algorithmic) prediction procedures: The clinical-statistical controversy. Psychology, Public Policy, and Law, 2, 293-323.

James, I. A., Blackburn, I. M., Milne, D. L., \& Reichfelt, F. K. (2001). Moderators of trainee therapists' competence in cognitive therapy. British Journal of Clinical Psychology, 40(2), 131-141.

Kahneman, D. (2013). Thinking, fast and slow. New York: Macmillan.

Kendall, P. C., Gosch, E., Furr, J. M., \& Sood, E. (2008). Flexibility within fidelity. Journal of the American Academy of Child \& Adolescent Psychiatry, 47(9), 987-993.

Kleinmuntz, B. (1990). Why we still use our heads instead of formulas: Toward an integrative approach. Psychological Bulletin, 107(3), 296. 
Lichtman, J. D. (2015). The case of "Hiro": Treating Tourette Syndrome by Comprehensive Behavioral Intervention for Tics (CBIT). Doctoral dissertation, Rutgers University.

Lichtman, J. D. (2017). The case of "Hiro": Treating Tourette Syndrome by Comprehensive Behavioral Intervention for Tics (CBIT). Pragmatic Case Studies in Psychotherapy, 13(1), Article 1, 1-37. Available: http://pcsp.libraries.rutgers.edu

Loeb, K. L., Wilson, G. T., Labouvie, E., Pratt, E. M., Hayaki, J., Walsh, B. T., Agras, W. S., \& Fairburn, C. G. (2005). Therapeutic alliance and treatment adherence in two interventions for bulimia nervosa: A study of process and outcome. Journal of Consulting and Clinical Psychology, 73, 1097-1106.

Nickerson, R. S. (1998). Confirmation bias: A ubiquitous phenomenon in many guises. Review of General Psychology, 2(2), 175.

Piacentini, J., Woods, D. W., Scahill, L., Wilhelm, S., Peterson, A. L., Chang, S., ... \& Walkup, J. T. (2010). Behavior therapy for children with Tourette disorder: A randomized controlled trial. Journal of the American Medical Association, 303(19), 1929-1937.

Ross, L. (1977). The intuitive psychologist and his shortcomings: Distortions in the attribution process. Advances in experimental social psychology, 10, 173-220.

Schulte, D., Künzel, R., Pepping, G., \& Schulte-Bahrenberg, T. (1992). Tailor-made versus standardized therapy of phobic patients. Advances in Behaviour Research and Therapy, 14(2), 67-92.

Specht, M. W., Woods, D. W., Nicotra, C. M., Kelly, L. M., Ricketts, E. J., Conelea, C. A., ... \& Walkup, J. T. (2013). Effects of tic suppression: ability to suppress, rebound, negative reinforcement, and habituation to the premonitory urge. Behaviour research and therapy, 51(1), 24-30.

Tversky, A. \& Kahneman, D. "This Week’s Citation Classic." Science 185 (1974): 1124-31.

Verdellen, C. W., Hoogduin, C. A., Kato, B. S., Keijsers, G. P., Cath, D. C., \& Hoijtink, H. B. (2008). Habituation of premonitory sensations during exposure and response prevention treatment in Tourette's syndrome. Behavior Modification, 32(2), 215-227.

Wellen, B, \& Himle, M.B. (2017). From cook to culinarian: Going beyond the manual when delivering behavior therapy to treat Tourette Disorder. Pragmatic Case Studies in Psychotherapy, 13(1), Article 3, 51-62. Available: http://pcsp.libraries.rutgers.edu

Wilson, G. T. (1998a). Manual- based treatment and clinical practice. Clinical Psychology: Science and Practice, 5(3), 363-375.

Wilson, G. T. (1998b). The clinical utility of randomized controlled trials. International Journal of Eating Disorders, 24(1), 13-29.

Wilson, G. T. (2007). Manual-based treatment: Evolution and evaluation. In T.A. Treat, R.R. Bootzin, \& T.B. Baker (Eds.). Psychological clinical science: Papers in honor of Richard M. McFall, 105-132. New York: Psychology Press.

Woods, D. W., Piacentini, J. C., Chang, S. W., Deckersbach, T., Ginsburg, G. S., Peterson, A. L., . . . Wilhelm, S. (2008). Managing Tourette syndrome: A behavioral intervention. Oxford: Oxford University Press. 